\title{
Intermittent Self-catheterization in Older Adults: Predictors of Success for Technique Learning
}

\begin{abstract}
Claire Hentzen ${ }^{1}$, Rebecca Haddad ${ }^{1}$, Samer Sheikh Ismael ${ }^{1}$, Benoit Peyronnet ${ }^{2}$, Xavier Gamé ${ }^{3}$, Pierre Denys ${ }^{4}$, Gilberte Robain ${ }^{5}$, Gérard Amarenco'; for the GRAPPPA* (Clinical research Group of perineal dysfunctions in older adults)
\end{abstract}

${ }^{1}$ Department of Neuro-Urology, Tenon Hospital, AP-HP, Paris, France

${ }^{2}$ Department of Urology, University Hospital of Rennes, Rennes, France

${ }^{3}$ Department of Urology, University Hospital of Toulouse, Toulouse, France

${ }^{4}$ Departement of Physical Medicine and Rehabilitation, Raymond Poincaré Hospital, AP-HP, Garches, France

${ }^{5}$ Departement of Physical Medicine and Rehabilitation, Rothschild Hospital, AP-HP, Paris, France

Purpose: The main goal of this retrospective study is to explore the predictors of success in learning clean intermittent selfcatheterization (CISC) in patients over 65 years of age. The secondary goal is to assess whether in this population, the risk of failure to perform CISC is greater, compared with patients under 65 with similar pathologies.

Methods: All patients older than 65 consulting between January 2011 and January 2016 for learning CISC were included. A control population younger than 65 matching with sex, body mass index, and pathology was selected.

Results: One hundred sixty-nine of the 202 patients $(83.7 \%)$ over 65 succeeded in learning CISC. Obesity $(\mathrm{P}<0.05)$, low pencil and paper test $(\mathrm{PP}$ test $)(\mathrm{P}<0.01)$ and low functional independence measure $(\mathrm{FIM})(\mathrm{P}<0.01)$ scores were risk factors of failure. No significant differences were found with sex or pathology. In multivariate analysis, low PP test perineum access (odds ratio [95\% confidence interval], 2.30 [1.32-4.42]), low FIM motor (1.04 [1.01-1.08]), and FIM cognition (1.18 [1.03-1.37]) scores were independent factors of learning failure. Compared to control group, age over 65 was not predictive of failure $(\mathrm{P}=0.15)$.

Conclusions: Our study shows that success in learning CISC does not depend on age but on difficulties in mobility, access to perineum and probably cognitive disorders.

Keywords: Intermittent urethral catheterization; Aged; Urinary retention; Urinary bladder diseases

- Ethics Research: This retrospective study was accepted by the local ethics committee; informed consent was not necessary. The study protocol and data collection were conducted in accordance with the ethical standards of the national research committee and with the Declaration of Helsinki.

- Conflict of Interest: GRAPPPA (Clinical research Group of perineal dysfunctions in older adults) was funded in 2013 by Prof. G. Amarenco. GRAPPPA is an independent group with no link with pharmaceutical industry concerning subject of research, study protocol and publication. Occasional logistical support can be asking. Except for that, no potential conflict of interest relevant to this article was reported.

\section{INTRODUCTION}

Clean intermittent self-catheterization (CISC) was introduced by Lapides et al. [1] in 1972. It is nowadays the gold standard for the treatment of urinary retention in neurogenic bladders [2]. But increasingly, CISC is prescribed to patients with nonneurogenic lower urinary tract symptoms (LUTS). Several studies have demonstrated an improvement in quality of life in

Corresponding author: Claire Hentzen (D) https://orcid.org/0000-0002-9223-4925 Department of Neuro-Urology, Tenon Hospital, 4 rue de la Chine, 75020 Paris, France

E-mail: claire.hentzen.mpr@gmail.com / Tel: +33-786481555 / Fax: +33-156017230

*The members of the GRAPPPA are listed in the ACKNOWLEDGEMENTS section.

Submitted: October 17, 2017 / Accepted after revision: March 1, 2018 
patients under CISC $[3,4]$. There are several indications for CISC in older adults, either in neurological condition (myelopathy following neck osteoarthritis, lumbar spinal stenosis, multiple sclerosis [MS], etc.) or not (bladder outlet obstruction, underactive detrusor [5], etc.).

Its effectiveness in prevention of infectious or renal complications, but also its safety, have been demonstrated, with a low risk of urinary tract infection [6,7]. Aseptic or clean intermittent catheterization (CIC) are taught to patients, with no evidence of the superiority of one over the other on the occurrence of complications. Guidelines on technique differ according to scientific societies.

However, due to factors associated with cognitive, motor, sensory, visual and even psychological disorders, healthcare providers are often reluctant to propose such a therapy in older patients, anticipating a learning failure. Very few studies have been conducted in this population to assess the feasibility and persistence of CISC [8-10].

The main objective of this study was to explore the predictors of success in learning CISC in patients over 65 years. The secondary goal was to assess whether in this population an increased risk of learning failure can be observed compared with patients under 65 years.

\section{MATERIALS AND METHODS}

This retrospective cohort study included all patients over 65 years, according to the MeSH (medical subject headings) definition of aged patient [11], seen in an outpatient Neuro-urology Department of a University Hospital for learning CISC between January 2011 and January 2016, regardless of their pathology. The indication of self-catheterization was generally carried out by the unit's doctors at a previous medical appointment. Patients were taught CISC during a day hospital, in which an occupational therapist and a neuro-psychologist assessments were performed. The occupational therapist assessed functional abilities using the functional independence measure (FIM) [12] (which is a good reflect of functional limitation in daily living activities). Ability to catheterize was estimated by the pencil and paper test (PP test) [13], a simple test performed in less than 4 minutes that included exercises of grip, access to perineum and cognitive tasks. Finally, patient learned and performed CISC technique with a continence nurse, according to a formal protocol of therapeutic education. It comprises anatomy explications with scheme or dummy, presentation of several models of catheter, trials of opening packaging, grip catheter and handling. Patient performed at least 2 trials of the technique, if possible on toilets, but it could be done on the wheelchair or on a bed. A medical evaluation concludes the day hospital to prescribe number of CISC by day and to adapt drug therapy if necessary. If patients failed after the first session, a second or third session could be proposed.

All data were collected retrospectively from medical records. Data initially collected were age, gender, etiology, weight, height, body mass index (BMI), FIM score, PP test score, if they lived alone or not. Conditions leading to LUTS were classified as neurological or nonneurological, central or peripheral nervous system diseases, and in 6 categories (MS, traumatic and nontraumatic spinal cord injury, cauda equina syndrome, congenital megacystis ["lazy bladder"], idiopathic, and others [which included suprapontine diseases and nonneurological diseases]). Missing data were not replaced.

Primary outcome was success or failure of this education (whether the patient could practice CISC or not). It was collected at the end of the training session. For those who had several sessions, the end of the last one was considered to define success or failure to catheterize. Potential predictors tested were age, gender, BMI and obesity, etiology of LUTS, functional abilities by FIM and PP test (total and subscores).

For secondary objective, a control population of subjects less than 65 years old, learning CISC during the same period and with a minimum follow-up of one month has been set, matching 1:1 with sex, pathology (based on the 6 categories previously mentioned) and BMI. Same data were collected. Outcome was success or failure to perform CISC after training session.

Statistical analyses were performed with the R software for Windows (Rx64 3.2.3, R Foundation for Statistical Computing, Vienna, Austria). Verification of normal distribution of all variables was done with Kolmogorov-Smirnov test. A chi-square test was performed to compare the qualitative variables or Fisher exact test when the expected number was less than 5. A 2-sample t-test was used for comparisons of quantitative variables or a Welch 2-sample t-test if variance was not equal. Statistical significance was set at the $95 \%$ level $(\mathrm{P}<0.05)$. A multivariate analysis using a logistic regression model was performed to seek for predictors of failure of CIC learning.

\section{RESULTS}

We enrolled 202 patients over 65 years (mean age, $73.7 \pm 6.2$ 
Table 1. Characteristics of study participants $(n=202)$

\begin{tabular}{lc}
\hline Characteristic & Value \\
\hline Age $($ yr $)$ & $73.6 \pm 6.2$ \\
Male sex & $77(38.1)$ \\
Living alone & $63(31.2)$ \\
Etiology of LUTS & \\
Multiple sclerosis & $42(20.8)$ \\
Cauda Equina Syndrome or neuropathy & $36(17.8)$ \\
Traumatic or nontraumatic spinal injury & $38(18.8)$ \\
Not determined & $29(14.4)$ \\
Congenital megacystis & $10(5.0)$ \\
Others & $46(22.8)$ \\
Comorbidities & \\
High blood pressure & $87(43.1)$ \\
Cardiopathy & $41(20.2)$ \\
Pulmonary disease & $35(17.3)$ \\
Nephrological disease & $14(6.9)$ \\
Diabetes & $28(13.9)$ \\
History of cancer & $48(23.8)$ \\
Treatment & \\
Polymedication $(\geq 5$ drugs) & $116(57.4)$ \\
Antipatelet agent & $56(27.7)$ \\
Anticoagulant & $14(6.9)$ \\
Antidepressant & $50(24.8)$ \\
\hline
\end{tabular}

Values are presented as mean \pm standard deviation or number (\%).

LUTS, lower urinary tract symptoms.

years) including 125 women (62\%) (Table 1). LUTS were neurogenic for 140 patients (69\%).

One hundred sixty-nine patients $(83.7 \%)$ successfully performed the technique (Table 2), 16 patients underwent 2 sessions ( $81.3 \%$ of success), 4 patients underwent 3 sessions ( $75 \%$ of success). Rate of patients who successfully completed learning according to age and sex is shown in Fig. 1. Coude tip catheter was useful for 3 patients, and 2 others needed specific tip ( 1 conical and 1 spherical, respectively).

A BMI over $30 \mathrm{~kg} / \mathrm{m}^{2}$, defining obesity, was associated with a failure to learn CISC $(\mathrm{P}=0.02)$. Patients who failed in learning had a lower FIM score $(\mathrm{P}=0.004)$. Composite FIM motor $(\mathrm{P}<0.001)$ and FIM cognition $(\mathrm{P}=0.043)$ scores were individually significant. Failure group had a lower $\mathrm{PP}$ test $(\mathrm{P}=0.009)$ score, especially for the item "access to perineum" $(\mathrm{P}=0.002)$ than success group. In contrast, items "cognition" and "prehension" were not statistically significant. Among patients over 65 years, the oldest were not at risk to fail.

In multivariate analysis (Table 3), several models were studied. In the first model with binary factors, obesity was a risk factor of failure $(\mathrm{P}=0.014$; odds ratio [95 confidence interval],
Table 2. Univariate analysis of factors associated with success of clean intermittent self-catheterization learning in patients over 65 years

\begin{tabular}{|c|c|c|c|}
\hline Variable & $\begin{array}{l}\text { Success } \\
(n=169)\end{array}$ & $\begin{array}{l}\text { Failure } \\
(\mathrm{n}=33)\end{array}$ & P-value \\
\hline Age $>75 \mathrm{yr}$ & $58(34.3)$ & $14(42.4)$ & $0.38^{\mathrm{a})}$ \\
\hline Mean \pm SD & $73.8 \pm 5.9$ & $74.8 \pm 7.4$ & $0.34^{\mathrm{c})}$ \\
\hline Female sex & $100(59.2)$ & $25(75.8)$ & $0.07^{\mathrm{a})}$ \\
\hline $\begin{array}{l}\text { Body mass index }>30 \mathrm{~kg} / \mathrm{m}^{2} \\
\text { Mean } \pm \mathrm{SD}\end{array}$ & $\begin{array}{r}17(10.2) \\
25.2 \pm 4.0\end{array}$ & $\begin{array}{c}9(29) \\
26.7 \pm 6.1\end{array}$ & $\begin{array}{l}0.019^{\mathrm{b})} \\
0.20^{\mathrm{d})}\end{array}$ \\
\hline Neurological disease & $114(67.4)$ & $26(78.8)$ & $0.20^{\mathrm{a})}$ \\
\hline Central NSD & $83(49.1)$ & $20(60.6)$ & $0.40^{\mathrm{a})}$ \\
\hline $\begin{array}{l}\text { Pathology } \\
\text { Multiple sclerosis } \\
\text { CES or neuropathy } \\
\text { T or NT spinal injury } \\
\text { Not determined } \\
\text { Congenital megacystis } \\
\text { Others }\end{array}$ & $\begin{array}{l}32(18.9) \\
30(17.6) \\
34(20.1) \\
26(15.4) \\
10(5.9) \\
36(21.3)\end{array}$ & $\begin{array}{c}10(30.3) \\
6(18.2) \\
4(12.1) \\
3(9.1) \\
0(0) \\
10(30.3)\end{array}$ & $0.33^{\mathrm{b})}$ \\
\hline $\begin{array}{l}\text { PP test } \\
\text { Total }(/ 15) \\
\text { Perineum }(/ 5) \\
\text { Cognition }(/ 5) \\
\text { Prehension }(/ 5)\end{array}$ & $\begin{array}{r}12.8 \pm 2.2 \\
4.7 \pm 0.7 \\
3.5 \pm 1.8 \\
4.7 \pm 0.7\end{array}$ & $\begin{array}{r}10.9 \pm 3.4 \\
3.6 \pm 1.5 \\
2.9 \pm 1.9 \\
4.3 \pm 1.3\end{array}$ & $\begin{array}{l}0.008^{\mathrm{d})} \\
0.002^{\mathrm{d})} \\
0.17^{\mathrm{c})} \\
0.12^{\mathrm{d})}\end{array}$ \\
\hline $\begin{array}{l}\text { FIM } \\
\text { Total (/126) } \\
\text { Cognition (/35) } \\
\text { Motor (/91) }\end{array}$ & $\begin{array}{c}108.2 \pm 14.8 \\
32.8 \pm 3.3 \\
75.4 \pm 13.2\end{array}$ & $\begin{array}{l}94.4 \pm 20.4 \\
30.4 \pm 5.5 \\
64.0 \pm 18.2\end{array}$ & $\begin{array}{l}0.004^{\mathrm{d})} \\
0.043^{\mathrm{d})} \\
<0.001^{\mathrm{c})}\end{array}$ \\
\hline
\end{tabular}

Values are presented as number (\%) or mean \pm standard deviation (SD).

NSD, nervous system disease, CES, Cauda Equina Syndrome; T or NT, traumatic or nontraumatic; PP test, pencil and paper test; FIM, functional independence measure.

${ }^{\text {a) }}$ Pearson chi-square test; ${ }^{\text {b) }}$ Fisher exact test; ${ }^{\text {c) }}$ Two-sample t-test; ${ }^{\text {d) }}$ Welch 2-sample t-test.

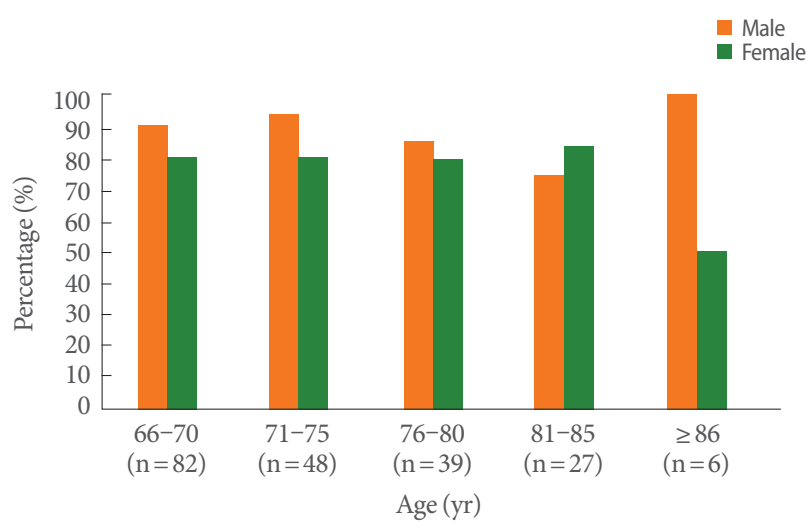

Fig. 1. Success rate according to age and sex. 
Table 3. Multivariable association between predictors and clean intermittent self-catheterization learning success

\begin{tabular}{lcccc}
\hline Variable & Model 1 & Model 2 & Model 3 & Model 4 \\
\hline Female sex & $0.49(0.19-1.12)$ & $0.13(0.07-1.26)$ & $0.15(0.03-0.50)^{* *}$ & $0.10(0.02-0.40)^{* *}$ \\
Obesity & $0.30(0.11-0.80)^{*}$ & $0.72(0.20-3.48)$ & $0.79(0.23-3.05)$ & $0.67(0.20-2.55)$ \\
Age $>75$ yr & $0.72(0.33-1.60)$ & $0.39(0.54-5.65)$ & $1.18(0.44-3.37)$ & $1.29(0.47-3.77)$ \\
Neurologic etiology & $0.5(0.19-1.21)$ & $0.22(0.05-1.58)$ & $0.67(0.17-2.30)$ & $0.7(0.18-2.41)$ \\
PP test perineum subscale & - & $2.30(1.32-4.42)^{* *}$ & - & - \\
PP test cognition subscale & - & $1.06(0.77-1.46)$ & - & - \\
PP test prehension subscale & - & $1.08(0.56-1.90)$ & - & - \\
FIM total & - & - & $1.05(1.02-1.08)^{* * *}$ & - \\
FIM cognition subscale & - & - & - & $1.18(1.03-1.37)^{*}$ \\
FIM motor subscale & - & - & - & $1.04(1.01-1.08)^{*}$
\end{tabular}

Values are presented as odds ratio (95\% confidence interval).

Model 1, multivariate analysis including binary factors; model 2, multivariate analysis including binary factors and items of PP test; model 3, multivariate analysis including binary factors and FIM score; model 4, multivariate analysis including binary factors and subscores of FIM score .

$\mathrm{PP}$ test, pencil and paper test; FIM, functional independence measure.

${ }^{*} \mathrm{P}<0.05 .{ }^{* *} \mathrm{P}<0.01 .{ }^{* *} \mathrm{P}<0.001$.

$0.30[0.11-0.80])$. In the second model including items of PP test, only the "access to perineum" item, remained significant $(\mathrm{P}=0.016 ; 2.30[1.32-4.42])$. Obesity was not significantly associated to failure, but acted as a confounder with the PP test "access to perineum" item $(\mathrm{P}=0.54)$. In the third model including FIM, female gender $(\mathrm{P}=0.006,0.15[0.03-0.50])$ and a low FIM total score $(\mathrm{P}<0.001,1.05[1.02-1.08])$ were factors associated with learning failure. The fourth analysis shows that low FIM motor and low FIM cognition scores are independent risk factors for failure.

Compared to control population under 65 years, older adults had lower PP test score $(\mathrm{P}<0.001)$ and lower FIM score $(\mathrm{P}<$ $0.001)$. No significant difference regarding failure or success to perform CISC $(\mathrm{P}=0.15)$ has been found, with 179 patients $(88.6 \%)$ able to perform the technique in control group. Number of CISC prescribed was not different: $38 \%$ of patients in the control group had a prescription for more than 3 CISC per day $(\mathrm{P}=0.74)$.

\section{DISCUSSION}

Our study, the first of its kind, shows that CISC learning is possible in older adults. In this cohort, limiting factors to perform the technique are functional and cognitive disabilities, perineum access difficulties, obesity and female gender.

Several limiting factors of CISC learning are usually mentioned: cognitive, motor difficulties, sensory disturbances, un- favorable anatomic factors, psychogenic and environmental factors. The therapeutic education programs include a clinical evaluation with generally achieving functional scores to assess patient's skills. No studies have been done specifically in older adults to assess learning opportunities. In the general population, very few studies focus on learning abilities [14,15], but rather on adherence to CISC.

Our study shows that obesity can negatively influence learning, probably related to abdominal apron, embarrassing access to the perineum. In women with spinal cord injury, ability to perform CISC is affected once BMI exceeded $25 \mathrm{~kg} / \mathrm{m}^{2}$ [16]. Moreover, in multivariate analysis, only the PP test item "access to perineum" remains significant, with a confounding effect of obesity. In fact, obesity is one of the variables that could restrict perineum access, especially in female patients in which length of the arms could be insufficient to position the catheter in front of the meatus due to abdominal apron, or in male patients with buried penis. Furthermore, obesity was no more significantly associated with the main outcome when FIM score was included in the multivariate model. One hypothesis is that decline in mobility may promote weight gain.

An assessment tool, the PP test [13], was established to assess, in a simply manner, the feasibility of CISC for patients, with a score of 0-5 for each item (grip strength, cognition, access to perineum). A low PP test score was obviously expected as a negative factor for learning CISC since it assesses the capacity to carry out this action. In contrast, a PP test score less 
than 10, even if it is unfavorable, should not contraindicate learning, as the minimum score in the success group was 7/15. Conversely, a score at 15 does not guarantee the success of learning.

Unsurprisingly, a low FIM score was also unfavorable to learning. While global, this tool is used to evaluate the functional abilities of patients. The score remained significantly associated with success for learning CISC in multivariate analysis, either on the motor or cognitive item, reflecting the need of intellectual in addition to pure motor skills for learning CISC. The items of PP test and FIM have deliberately not been submitted within the same analysis as these two tests evaluate functional capacities, with consequent redundancy.

No significant difference related to gender was found in univariate analysis, and then though, intuitively, perineum in women seems to be more difficult to access. This is possibly related to a lack of power of this study, sex appearing statistically significant in multivariate analysis.

However, some limitations restrict our study's interpretation. First it was a single-center retrospective study, with no control group and the present report is flawed by the biases inherent to this study design. The initial assessment by a neurourologist probably induced bias in initial patient selection, since those whose learning is clearly impossible are not referred to dayhospital to attempt CISC learning. Thus, this bias is supposed to be similar with the younger patient. The choice of 65 age limit is debatable. It is the most widely used the definition in literature for aged patient [11] while the World Health Organization keep proposing 60 years old as a threshold. Currently the notion of "physiological age" is usually preferred [17]. Unfortunately, the study design did not permit to classify the patient with geriatric scale such as Cumulative Illness Rating Scale [18].

In our study, neurological disorders are not a limiting factor to CISC learning. This can be explained by the diversity of neurological pathologies, leading to very different levels of disability. Inclusion of all patients, regardless of their pathology, constructed a heterogeneous population. Other comorbidities in nonneurological patients can also cause motor (rheumatic diseases) or cognitive (iatrogenic) impacts.

Concerning cognitive disorders, these can be induced by the responsible disease (MS), or be linked to other disease (dementia for example). In a previous study on patients with MS, cognitive impairment (assessed by Brief Repeatable Battery of Neuropsychological Tests) does not seem to influence the capacity to learn the technique [14]. Many tests are available to assess cognition, sometimes not validated in all pathologies.
Absence of recommendations involves various tools used across centers. Though, rationality of cognitive limitation to perform CISC seems evident, since the technique includes schedule and memorization tasks. Our study objectives role of patient's cognitive abilities, rate by the composite score FIM cognition, but not on cognition item of PP test, probably less specific.

Visual disturbances, more common in older population (cataract, macular degeneration, etc.), may complicate learning. However, a blind patient in the study of Whitelaw et al. [8] had managed learning. The study design did not permit us to assess the impact of visual disturbances in our cohort.

Upper limb at least partially functional are important for installation (transfer, undressing), and for the technique itself. Vahter et al. [14] found in patient with MS a correlation between learning failure and an Expanded Disability Status Scale (EDSS), reflecting patient's low mobility capabilities. For quadriplegics, it is agreed that the limit required by the classification Zancolli is C6A/C5B [19], and sometimes patients need a continent catheterizable stoma. But physical disabilities are not insurmountable. In one of the few studies in older adults, a patient with only one functional upper limb was able to perform CISC [8]. Despite digital osteoarthritis with low dexterity in $10 \%$ of patients in the study of Bennett and Diokno [9], they all had acquired the technique. Ways of assessment are varied, and depend on a part of the patient's condition: EDSS in MS [20], American Society Injury Association in spinal cord injured [21], but also on what we want to assess. Prehension can be evaluated by Nine Hole Peg Test [22], or by Action Research Arm Test [23]. Spasticity assessment of adductors can be done using the modified Ashworth scale [24]. Finally, transfer capabilities can be evaluated by some items of motor FIM. Our study did not evaluate upper limb function with a specific scale because of time constraint during CISC education. The PP Test assesses prehension with similar actions necessary for CISC, and access to perineum. The FIM score by motor items assesses partially upper limb function, and is statistically correlated with Fugl-Meyer scale [25].

Disturbances of sensation can also interfere. Anesthesia of the perineal area in patients with visual disturbances will make learning difficult, since neither viewing the mirror, nor tracking the touch will be easy. The evaluation is done by neurologic examination of the perineum. Similarly, sensory disorders in the upper limbs make it more difficult to grip the catheter or to spread labia in women to expose the meatus.

Anatomically, difficulties to reach the meatus may compli- 
cate learning, as buried, ectopic, distal urethral orifice and even hypospadias urethra. In addition, women, especially old women, often do not have a clear knowledge of their perineum anatomy; many say "they never have looked at this part of their body." In men, a prostate hypertrophy can block catheter insertion by creating an obstacle to the passage. Using Coude or Tieman tip catheter generally overcomes this obstacle and facilitates CISC.

There are many environmental factors. It is demonstrated that learning in a reference center with a standardized patient education program improves chances of success [26]. Then, the space environment at home, for example the presence of sink in the bathroom or the ease of access to toilets, may interfere with the pursuit of CISC. As a tertiary referral center, long-term follow-up for CISC was made in several times in local institutions. Adherence rate and complication as infections or urethral injuries were not systematically available.

Moreover, psychogenic factors may also influence learning. The missing data of neuropsychological assessment did not permit us to use these for statistical analysis which could be regarded as a shortcoming due to the well-established impact on this factor on CISC learning. Transition to CISC may represent a new loss of function for the patient, even if he already knows the abnormal functioning of his urinary tract. Several feelings were described by patients: the feeling of loss and recovery of function failure, anxiety, lack of knowledge about CISC, stigma and negative associations, embarrassment about very intimate learning, etc.[27]. Neuropsychological assessment also evaluates the patient's mental state and his feelings about CISC. To best reduce the negative psychogenic factors, a methodological guide complies with HAS (Haute Autorité de Santé in France) recommendations was implemented for learning CISC [28].

Finally, age, does not affect learning success, showing that decision to prescribe CISC must be influenced on functional skills and not on age. Thus, Parsons et al. [29] on a study of 309 nonneurological patients, did not put any evidence of influence of age or sex on adherence to CISC at 6 weeks, while another study showed a lower adherence at 1-year follow-up in patients over 60 years [30].

In conclusion, this study shows that older age is not a risk factor for failure in learning of self-catheterize. Less functional and cognitive abilities (FIM, PP test), and perineum access difficulties may favor the failure of learning. Severe disabilities do not always result in unsuccessful learning. If the presence of one unfavorable factor could not reject the patient to try the technique, probably accumulation of them is a good predictor of failure. Follow-up of these patients will track down factors affecting adherence to CISC at short and medium term.

\section{ACKNOWLEDGEMENTS}

The members of GRAPPPA (Clinical research Group of perineal dysfunctions in older adults): Gérard Amarenco, Xavier Deffieux, Brigitte Fatton, Xavier Gamé, Rebecca Haddad, Claire Hentzen, Claude Jeandel, Jacques Kerdraon, Pierre Mares, Matthieu Mezzadri, Anne Claire Petit, Benoit Peyronnet, Gilberte Robain, Jean Marc Vetel.

\section{AUTHOR CONTRIBUTION STATEMENT}

- Full access to all the data in the study and takes responsibility for the integrity of the data and the accuracy of the data analysis: C. Hentzen, G. Amarenco

-Study concept and design: C. Hentzen, B. Peyronnet, X. Gamé, P. Denys, G. Robain, G. Amarenco

- Acquisition of data: C. Hentzen, G. Amarenco, R. Haddad

- Analysis and interpretation of data: C. Hentzen, R. Haddad, B. Peyronnet, X. Gamé, P. Denys, G. Robain, G. Amarenco

- Drafting of the manuscript: C. Hentzen, R. Haddad, S. Sheikh

\section{Ismael, G. Amarenco}

-Critical revision of the manuscript for important intellectual content: G. Amarenco, G. Robain, X. Gamé

- Statistical analysis: C. Hentzen, R. Haddad

- Administrative, technical, or material support: G. Amarenco

-Study supervision: G. Amarenco

\section{REFERENCES}

1. Lapides J, Diokno AC, Silber SJ, Lowe BS. Clean, intermittent selfcatheterization in the treatment of urinary tract disease. J Urol 1972;107:458-61.

2. Stöhrer M, Blok B, Castro-Diaz D, Chartier-Kastler E, Del Popolo G, Kramer G, et al. EAU guidelines on neurogenic lower urinary tract dysfunction. Eur Urol 2009;56:81-8.

3. Bjerklund Johansen T, Hultling C, Madersbacher H, Del Popolo G, Amarenco G; LoFric Primo Study Group. A novel product for intermittent catheterisation: its impact on compliance with daily life-international multicentre study. Eur Urol 2007;52:213-20.

4. Bakke A, Malt UF. Social functioning and general well-being in patients treated with clean intermittent catheterization. J Psychosom 
Res 1993;37:371-80.

5. van Koeveringe GA, Rademakers KL, Birder LA, Korstanje C, Daneshgari F, Ruggieri MR, et al. Detrusor underactivity: pathophysiological considerations, models and proposals for future research. ICI-RS 2013. Neurourol Urodyn 2014;33:591-6.

6. Hunt GM, Oakeshott P, Whitaker RH. Intermittent catheterisation: simple, safe, and effective but underused. BMJ 1996;312:103-7.

7. Giannantoni A, Scivoletto G, Di Stasi SM, Silecchia A, FinazziAgrò E, Micali I, et al. Clean intermittent catheterization and prevention of renal disease in spinal cord injury patients. Spinal Cord 1998;36:29-32.

8. Whitelaw S, Hammonds JC, Tregellas R. Clean intermittent selfcatheterisation in the elderly. Br J Urol 1987;60:125-7.

9. Bennett CJ, Diokno AC. Clean intermittent self-catheterization in the elderly. Urology 1984;24:43-5.

10. Pilloni S, Krhut J, Mair D, Madersbacher H, Kessler TM. Intermittent catheterisation in older people: a valuable alternative to an indwelling catheter? Age Ageing 2005;34:57-60.

11. Aged-MeSH-NCBI [Internet]. Bethesda (MD): National Center for Biotechnology Information, U.S. National Library of Medicine; [cited 2017 Dec 18]. Available from: https://www.ncbi.nlm.nih.gov/ mesh/68000368.

12. Ottenbacher KJ, Hsu Y, Granger CV, Fiedler RC. The reliability of the functional independence measure: a quantitative review. Arch Phys Med Rehabil 1996;77:1226-32.

13. Amarenco G, Guinet A, Jousse M, Verollet D, Ismael SS. Pencil and paper test: a new tool to predict the ability of neurological patients to practice clean intermittent self-catheterization. J Urol 2011; 185:578-82.

14. Vahter L, Zopp I, Kreegipuu M, Kool P, Talvik T, Gross-Paju K. Clean intermittent self-catheterization in persons with multiple sclerosis: the influence of cognitive dysfunction. Mult Scler 2009; 15:379-84.

15. Brown JP, Reichenbach MB. Screening children with myelodysplasia for readiness to learn self-catheterization. Rehabil Nurs 1989; 14:334-7.

16. Zlatev DV, Shem K, Elliott CS. How many spinal cord injury patients can catheterize their own bladder? The epidemiology of upper extremity function as it affects bladder management. Spinal Cord 2016;54:287-91.

17. Lixie E, Edgeworth J, Shamir L. Comprehensive analysis of large sets of age-related physiological indicators reveals rapid aging around the age of 55 years. Gerontology 2015;61:526-33.
18. Parmelee PA, Thuras PD, Katz IR, Lawton MP. Validation of the cumulative illness rating scale in a geriatric residential population. J Am Geriatr Soc 1995;43:130-7.

19. Asayama K, Kihara K, Shidoh T, Shigaki M, Ikeda T. The functional limitations of tetraplegic hands for intermittent clean self-catheterisation. Paraplegia 1995;33:30-3.

20. Kurtzke JF. Rating neurologic impairment in multiple sclerosis: an expanded disability status scale (EDSS). Neurology 1983;33:144452.

21. El Masry WS, Tsubo M, Katoh S, El Miligui YH, Khan A. Validation of the American Spinal Injury Association (ASIA) motor score and the National Acute Spinal Cord Injury Study (NASCIS) motor score. Spine (Phila Pa 1976) 1996;21:614-9.

22. Mathiowetz V, Weber K, Kashman N, Volland G. Adult norms for the nine hole peg test of finger dexterity. OTJR Occup Particip Health 1985;5:24-38.

23. Lyle RC. A performance test for assessment of upper limb function in physical rehabilitation treatment and research. Int J Rehabil Res 1981;4:483-92.

24. Pandyan AD, Johnson GR, Price CI, Curless RH, Barnes MP, Rodgers $\mathrm{H}$. A review of the properties and limitations of the Ashworth and modified Ashworth Scales as measures of spasticity. Clin Rehabil 1999;13:373-83.

25. Shelton FD, Volpe BT, Reding M. Motor impairment as a predictor of functional recovery and guide to rehabilitation treatment after stroke. Neurorehabil Neural Repair 2001;15:229-37.

26. Oh SJ, Ku JH, Lim SH, Jeon HG, Son H. Effect of a 'centralized intensive education system' for clean intermittent self-catheterization in patients with voiding dysfunction who start catheterization for the first time. Int J Urol 2006;13:905-9.

27. Ramm D, Kane R. A qualitative study exploring the emotional responses of female patients learning to perform clean intermittent self-catheterisation. J Clin Nurs 2011;20:3152-62.

28. Amarenco G. Guide méthodologique déducation thérapeutique du patient à l'autosondage [Internet]. [cited 2016 Apr 23]. Available from: http://www.sofmer.com/download/etp-as-final.pdf

29. Parsons BA, Narshi A, Drake MJ. Success rates for learning intermittent self-catheterisation according to age and gender. Int Urol Nephrol 2012;44:1127-31.

30. Girotti ME, MacCornick S, Perissé H, Batezini NS, Almeida FG. Determining the variables associated to clean intermittent selfcatheterization adherence rate: one-year follow-up study. Int Braz J Urol 2011;37:766-72. 